\title{
BMJ Open Mixed methods evaluation of a hospital group model using an embedded research approach: study protocol
}

\author{
Cecilia Vindrola-Padros, ${ }^{\oplus 1}$ Estela Capelas Barbosa, ${ }^{\oplus 1}$ Angus I G Ramsay, ${ }^{1}$ \\ Simon Turner, ${ }^{2}$ Stephen Morris, ${ }^{1}$ Ronald Agble, ${ }^{3}$ Amy Caldwell-Nichols, ${ }^{3}$ \\ Naomi J Fulop ${ }^{\oplus 1}$
}

To cite: Vindrola-Padros C, Capelas Barbosa E,

Ramsay AIG, et al. Mixed methods evaluation of a hospital group model using an embedded research approach: study protocol. BMJ Open 2019;9:e027086. doi:10.1136/ bmjopen-2018-027086

- Prepublication history for this paper is available online. To view these files, please visit the journal online (http://dx.doi. org/10.1136/bmjopen-2018027086).

Received 10 0ctober 2018 Revised 14 March 2019 Accepted 21 May 2019

A Check for updates

(C) Author(s) (or their employer(s)) 2019. Re-use permitted under CC BY-NC. No commercial re-use. See rights and permissions. Published by BMJ.

${ }^{1}$ Department of Applied Health Research, University College London, London, UK

${ }^{2}$ School of Management, University of Los Andes, Bogota, Colombia

${ }^{3}$ Royal Free London NHS Foundation Trust, London, UK

Correspondence to Dr Cecilia Vindrola-Padros; c.vindrola@ucl.ac.uk

\section{ABSTRACT}

Introduction Hospital group models represent an organisational form that aims to bring together multiple provider organisations with a central headquarters and unified leadership responsible for locally managed operating units, standardised systems and a value-set shared across the group. These models seek to improve outcomes by reducing unwarranted variations in care provision and reducing costs through economies of scale. There is limited evidence on the impact and processes of implementing these models, so this study aims to evaluate one case study of a hospital group model.

Methods and analysis We will conduct a formative, mixed-methods evaluation using an embedded research approach to analyse the implementation of the model and its impact on outcomes and costs. We will carry out a multisited ethnography to analyse the programme theory for model design and implementation, the barriers and facilitators in the implementation; and wider contextual issues that influence implementation using semistructured interviews $(n=80)$, non-participant observations ( $\mathrm{n}=80$ hours), 'shadowing' ( $\mathrm{n}=20$ hours) and documentary analysis. We will also carry out an economic evaluation composed of a cost-consequence analysis and a return on investment analysis to evaluate the costs of creating and running the model and balance these against the potential cost-savings.

Ethics and dissemination The study protocol was reviewed by the local R\&D Office and University College London Ethics Committee and classified as a service evaluation, not requiring approval by a research ethics committee. We will follow guidelines for informed consent, confidentiality and information governance, and address issues of critical distance prevalent in embedded research. Findings will be shared at regular time points to inform the implementation of the model. The evaluation will also generate: an evaluation framework to evaluate future changes; recommendations for meaningful baseline data and measuring improvement; identification of implementation costs and potential cost-savings; and lessons for the National Health Service on implementing these models.

\section{INTRODUCTION}

Several countries are currently implementing models of healthcare delivery that rely on

\section{Strengths and limitations of the study:}

- The embedded approach aims to facilitate the co-design of the study with research users and regular sharing of evaluation findings while maintaining a critical distance.

- The multisited ethnography will enable the exploration of implementation processes and experiences of the model from the perspectives of a wide range of stakeholders and multiple participating sites.

- The economic component of the evaluation will compare the group model with non-group model comparators, allowing for broader lessons for the National Health Service.

- This is an evaluation of one hospital group model, thus limiting the generalisability of the findings.

- The evaluation is dependent on the implementation of the group model within the study time frame, which creates a risk for the study if there are implementation delays or if the implementation does not occur.

joint-working arrangements between multiple healthcare providers in the form of hospital groups or chains. ${ }^{1}$ In England, the Five Year Forward View sets out a future strategy for the National Health Service (NHS) through the implementation of new care models. ${ }^{2} \mathrm{~A}$ review of new organisational models for delivering care in the NHS also presented models through which providers could develop formal arrangements to collaborate and work at scale, ${ }^{3}$ thereby improving outcomes by reducing unwarranted variations in care provision and reducing costs through economies of scale. A 'hospital group' has been defined as an organisational model that brings together multiple provider organisations and has the following characteristics: (1) a central headquarters responsible for unified leadership; (2) locally managed operating units; (3) standardised systems, practices and protocols; and (4) a culture and value-set that is shared across the group. ${ }^{3-5}$ Four group models have 
Table 1 Types of hospital group models in England

\begin{tabular}{|c|c|c|}
\hline Model & Description & Legal form \\
\hline 1. Federation & $\begin{array}{l}\text { Organisations come together to collaborate } \\
\text { on areas of mutual interest, after agreeing } \\
\text { on a set of common standards. Each } \\
\text { organisation retains its sovereignty }\end{array}$ & $\begin{array}{l}\text { Agreement can be set out in a legal } \\
\text { contract or memorandum of understanding }\end{array}$ \\
\hline 2. Delegated authority & $\begin{array}{l}\text { Organisations agree to delegate some or all } \\
\text { decision-making to a single organisation. } \\
\text { Each organisation retains its sovereignty and } \\
\text { the decision-making body is accountable to } \\
\text { the Boards of each organisation }\end{array}$ & $\begin{array}{l}\text { Trusts can set-up their own committees } \\
\text { (with the same remit and membership), } \\
\text { which can be supported by a legally } \\
\text { binding contractual joint venture between } \\
\text { the providers }\end{array}$ \\
\hline 3. Management responsibility & $\begin{array}{l}\text { One organisation takes management } \\
\text { responsibility for another. Each organisation } \\
\text { retains its Board, but the host organisation } \\
\text { is accountable for the performance of } \\
\text { organisations under its management }\end{array}$ & $\begin{array}{l}\text { The host organisation enters into a } \\
\text { management contract with another } \\
\text { organisation for an agreed period of time }\end{array}$ \\
\hline
\end{tabular}

Source, Based on NHS Group Models developed by Credo Business Consulting. ${ }^{4}$

NHS, National Health Service.

been accredited by NHS Improvement. These hospital groups have acquired distinct organisational models and legal forms ${ }^{4}$ (table 1).

\section{A case study of a group model}

This evaluation focuses on one case study of a hospital group model (type 4, model with different types of membership and organisational sovereignty listed in table 1). The aim of this organisational form is to introduce new ways of operating across a group of providers. Providers can join the group as full members (losing sovereignty to the group headquarters) or clinical partners (maintaining sovereignty and selecting which group model services to participate in). The case study we are evaluating includes one full member organisation formed in a merger of two organisations in 2014 (three hospital sites in total), and two clinical partners (one organisation with one hospital site and another with three hospital sites). Anticipated benefits of the implementation of this group model are: (1) improve clinical outcomes and increase patient safety and satisfaction by reducing unwarranted variations in care, (2) improve staff experience through career development opportunities and (3) reduce unit and system costs by streamlining wasteful processes and through economies of scale. ${ }^{6}$ To achieve this, the group aims to standardise clinical and non-clinical processes, consolidate clinical, clinical support and non-clinical services, and develop processes to promote leadership and workforce development (table 2). ${ }^{6}$

Group members will be connected through common processes, governance and back-office systems, led by a group headquarters. The group model structure will comprise a series of hospital units which will report to a Hospital Group Board. Clinical Working Groups (CWGs) will work across all hospital units. Each CWG contains a number of pathways that will be redesigned and standardised across relevant hospital units to eliminate unwarranted variation in care. Shared services, in the form of clinical support (eg, radiology and pharmacy) and non-clinical services (eg, finance and HR), will be centralised and provide support to all hospital units with full or clinical partner membership.

\section{Overall approach to the study}

We will use an embedded research approach, ${ }^{78}$ where researchers who have dual affiliation (to an academic institution and a healthcare organisation) are 'embedded' within the organisation(s) and co-produce knowledge with local staff that responds to the needs of the organisation and helps build research capacity. ${ }^{7}$ The principle of co-production is a core element of embedded research and it is based on the premise that researchers and members of staff from various layers and areas of the organisation can all provide different types of useful knowledge and expertise. ${ }^{7}$ In the case of this evaluation, we will collaborate closely with staff and managers from the healthcare organisations, to co-design and conduct research on identified needs, and share formative feedback at regular intervals. The embedded approach will also allow us to adapt rapidly to changes in context and make modifications in the study design, as appropriate while maintaining the independence of the research. 
Table 2 Objectives, processes and expected outcomes of the hospital group model

\section{Objective \\ 1. Reduce unwarranted variation in}

clinical and non-clinical processes

2. Consolidate clinical and non-clinical activity by centralising and removing duplication of services
Process

- Implement evidence-based standard clinical processes

- Standardise approach to non-clinical processes

- Centralise non-clinical activity

- Consolidate clinical support services across the group

- Consolidate clinical services to drive quality and value
3. Use leadership and expertise to drive quality improvement

Invest in leadership capabilities and workforce development

- Promote the better use of resources across the group

- Effective performance management of members

$\begin{aligned} & \text { 4. Deliver system-wide benefits through } \\ & \text { whole pathway re-design }\end{aligned}$
providers

\section{Outcome}

- Improve clinical outcomes and reduce unit and system-wide cost

- Deliver cost savings and free up clinician time

- Improve quality and reduce cost

- Generate economies of scale, improve the use of specialist resources and improve service quality

- Improve quality, effectiveness and reduce the cost of delivery (eg, by reducing the duplication of services across organisations)

- Staff with skills and expertise to deliver transformation

- Improve use of resources across the group using increase scale Improve performance and enable the delivery of transformation

- Reduce admissions to secondary care, and thereby reduce total system costs; improve patient outcomes and satisfaction

Source: Greyed out row (deliver system-wide benefits through whole pathway re-design) is outside scope of this evaluation as changes will not be implemented within the time scale of the evaluation.

The study protocol was co-produced and the selection of areas to be included in the evaluation was informed by:

- Early engagement with teams in the lead healthcare organisation in 2016.

- Meetings with teams in the lead healthcare organisation to discuss developments in group priorities and participate in a bidders' event (June to September 2017).

- Scoping exercise and prioritisation workshop to prioritise areas for the evaluation (October to November 2017).

\section{Research questions}

The evaluation will address the following research questions:

1. What is the programme theory underpinning the group model and how does it develop over time?

2. What are the processes used to implement organisational change across the group and how do these develop over time?

3. What are the barriers and facilitators encountered in the implementation of the group model? What is the impact of wider contextual factors?

4. What quantifiable impacts, costs, potential benefits and cost-savings, if any, does the group model have within different contexts?

5. What are the lessons for implementing models of this kind?

\section{METHODS AND ANALYSIS \\ Design}

The study will use a mixed-methods design, combining multisited ethnographic research with a health economics evaluation to evaluate a case study of a hospital group model.

The evaluation will focus on five main areas of the hospital group model (table 3). These areas will be used as 'tracer issues'. ${ }^{9}$ The evaluation will trace the activities of pathway teams within two Clinical Working Groups, including an analysis of specific tasks, processes for establishing evidence-based standards and measurement processes; engagement with the new e-platform (digitisation), and interaction between different organisations within the group structure. It will also look at how approaches to implementing the new structure might enable or hinder implementation success (identified as the capacity of the group model to achieve its goals of standardisation and consolidation). The evaluation will examine the development of the quality improvement and leadership development programmes (eg, main activities, staff perceptions and use of the programmes, potential impact of the programmes) across the hospital group. The study will also analyse the consolidation of clinical support services (pharmacy) and non-clinical services (finance). The evaluation will analyse the original plans behind the design of the hospital group model and how these have changed through time. An important component of the evaluation will be capturing the experiences of 
Table 3 Main areas included in the evaluation

\begin{tabular}{|c|c|c|c|}
\hline Programme/Project area* & Sub-areas $†$ & Site coverage & Implementation timeline \\
\hline 1. Group operating model & Governance & $\begin{array}{l}\text { All organisations currently } \\
\text { within the group and new } \\
\text { organisations joining the group }\end{array}$ & $\begin{array}{l}\text { Started planning the group } \\
\text { model with three existing sites } \\
\text { in August 2017, with additional } \\
\text { sites joining as clinical partners }\end{array}$ \\
\hline $\begin{array}{l}\text { 2. Two pathways within two } \\
\text { CWGs }\end{array}$ & $\begin{array}{l}\text { Two pathways (within each } \\
\text { CWG): } \\
\text { 1. Induction of labour (Women } \\
\text { and Children's CWG) } \\
\text { 2. Heart failure (Medicine and } \\
\text { Urgent Care CWG) }\end{array}$ & $\begin{array}{l}\text { All organisations currently } \\
\text { within the group and new } \\
\text { organisations joining the group } \\
\text { (pathways cover all sites) }\end{array}$ & $\begin{array}{l}\text { Piloting of pathways started } \\
\text { in January } 2018 \text {. Induction of } \\
\text { labour has implemented the } \\
\text { changes in two organisations. } \\
\text { Heart failure is still in } \\
\text { the piloting stage }\end{array}$ \\
\hline 3. Corporate Support Services & Finance & $\begin{array}{l}\text { All organisations currently } \\
\text { within the group and new } \\
\text { organisations joining the group }\end{array}$ & $\begin{array}{l}\text { Implementation date to be } \\
\text { determined }\end{array}$ \\
\hline
\end{tabular}

${ }^{*}$ Processes of joining the group will be captured in all areas.

†Staff experience impacts will be explored qualitatively in all areas.

CWG, Clinical Working Group; QI, quality improvement.

the local units who are part of the group, from the initial stages of joining the group through full implementation (as allowed by the study time frame).

\section{Implementation study of the group model}

We will carry out an organisational study of the implementation of this group programme using a multisited ethnographic approach. Multisited ethnographies allow researchers to capture different views, interactions and communication across a wide range of contexts. ${ }^{10}$ Adopting characteristics of a more 'mobile ethnography, ${ }^{11}$ multisited ethnographies facilitate the identification of changes in processes across physical spaces (ie, different healthcare environments) and conceptual spaces (ie, clinical pathways).

This component of the evaluation aims to use embedded research to understand how the group model is being governed and implemented in real time and how it changes over time, to facilitate more effective implementation, identify lessons for enabling improvement and inform the health economic component of the study. We will explore three main dimensions of the implementation of the group programme: (1) changes in organisational structures (eg, Boards, committees and local hospital units), (2) changes in service organisation and delivery and (3) quality improvement and leadership development. This will involve studying (a) the development and implementation of the new organisational structures, processes and cultures associated with the group model and (b) gathering stakeholders' (including clinical, non-clinical and management staff from across sites) perceptions of the group model and processes of implementing change within the healthcare organisations, including staff members' views on their engagement with the model.

\section{Economic evaluation}

We will investigate the costs, the potential cost savings, and the potential benefits of implementation of the hospital group model.

We expect the implementation of the group model to produce set-up costs and additional running costs. The set-up costs are likely to include direct and/or opportunity costs related to staff (training and change in staff-patient ratio) and capital (cost of digitisation, cost of IT system upgrade and so on), whereas the additional running costs are likely to refer to increased administrative workload (eg, additional group meetings, board meetings and so on). Benefits and impacts will be measured consistently with the process evaluation (processes defined in table 2). As the outputs from these consist mostly of process measures, a meaningful quantitative ranking will be derived from the qualitative ethnographic findings.

One important issue is the perspective of the analysisthe institutional viewpoint to take when measuring costs, cost-savings and benefits. From a health economics point of view, three separate and relevant perspectives exist: (1) the Hospital Group; (2) full members or clinical partners and (3) CWGs running across partners. The most relevant perspective for the evaluation should be jointly decided with local teams, following the same principle of co-production already outlined above. 
Table 4 Economic approach chosen for each component of the evaluation

\begin{tabular}{ll}
\hline Programme/Project area & Economic approach \\
\hline Group operating model & CCA \\
$\begin{array}{l}\text { Clinical Practice Groups } \\
\text { (CWGs) }\end{array}$ & CCA and ROI \\
$\begin{array}{l}\text { Leadership and Workforce } \\
\text { Capability }\end{array}$ & CCA framework \\
\hline
\end{tabular}

CCA, cost-consequence analysis; CWG, Clinical Working Group.; ROI, return on investment.

Based on the priorities identified by local staff in interviews from the scoping phase of the evaluation and documentary analysis, we are proposing two economic approaches. The first is a cost-consequence analysis (CCA). A CCA is a form of economic evaluation comparing alternative interventions in which the components of incremental costs (direct or indirect) and consequences (eg, knowledge, behaviours and processes) are computed and listed, without aggregating these results into a cost-effectiveness ratio. The CCA has been previously described as distinct from both cost-effectiveness analysis (CEA) and cost-benefit analysis (CBA). ${ }^{12}$ This approach was selected as it enables producing quantifiable results from process measures and other findings traditionally evaluated qualitatively, such as quality improvement (QI). The CCA will rely on emerging findings from the organisational study in order to define quantifiable consequence parameters, such as the degree of standardisation of pathways or number of leaders trained within the group model.

The second economic approach is a return on investment (ROI) study. This will allow for the costs of the model to be balanced against potential cost-savings that relate to the decrease in unwarranted variation (CWGs: two pathways). This second component of the economic evaluation will be carried out in addition to the CCA within the 30-month time scale (see table 4). Unlike the CCA, the ROI analysis does not depend or rely on the qualitative organisational study.

Both the CCA and ROI analysis will take an incremental approach, which implies defining a counterfactual or a comparator, which could be another hospital group or the extrapolation of the performance of each site composing the group model before they joined the group. The comparator ought to be jointly defined with the hospital group stakeholders via the formative feedback process established by the embedded research team.

Although ideally the same areas covered in the organisational component of this study would be evaluated in economic terms, due to implementation delays or data unavailability, the economic evaluation will focus on the Group operating model and the two pathways of the clinical practice groups as originally planned. Using the embedded approach as an opportunity for research capacity development, it was agreed between the research team and group stakeholders that area of Leadership and Workforce capability would produce a CCA framework, to be carried out at a later stage after this research.

\section{Data collection}

Implementation study of the group model

Data collection will be carried out through semi-structured interviews, non-participant observation and documentary analysis. Following a multisited approach, researchers will consider data collection as a mobile process, entailing physical presence in multiple organisations and conceptual analysis of various social spaces. ${ }^{14}$ In order to ensure the depth and richness of data despite the breadth of organisational coverage, we will use a 'focused ethnography' approach, which is based on targeted fieldwork in a series of locations over short periods of time. ${ }^{14}$

We will analyse the experiences of local sites by documenting processes of joining the group and different stages of implementation of the programmes of the group model. Data collection will focus on the three dimensions outlined above (changes in organisational structures and cultures, service organisation and delivery, and leadership development and quality improvement). Semi-structured interviews will be conducted at multiple organisational levels within the group structure and also capture the perceptions of member Trusts and other local and external stakeholders (table 5). Repeat interviews will be carried out with a sub-section of those interviewed in round 1 to enable a longitudinal analysis of programme implementation. Topic guides will be used to guide the interviews and the interviews will be audio recorded.

Following the tracer issues (table 3), the evaluation team will observe relevant planning and implementation meetings at group level (eg, Hospital Group Board meetings), CWG level (eg, CWG implementation group meetings), at the level of hospital units (eg, Board meetings), divisions (eg, committee meetings) and clinical team level (eg, pathway team meetings). We will aim to shadow the pathway leads to understand how their daily activity spans multiple organisations (up to 20 hours). We will aim to observe training sessions from the QI and leadership development programmes and related leadership events. We will also observe project management meetings (covering the same levels) used to plan the implementation of the consolidation of clinical support and non-clinical services. The observations will be documented in the form of field notes.

Documents from the various areas included in the evaluation will also be collected. These will include reports, planning documents and minutes from the meetings outlined above. These will be collected from the area leads and meeting Chairs. A document inventory will be used to catalogue the documents.

\section{Economic evaluation}

For the CCA analysis, two types of cost data will be required. First, outgoings and investments, both in terms of setting up the group model and running it on 


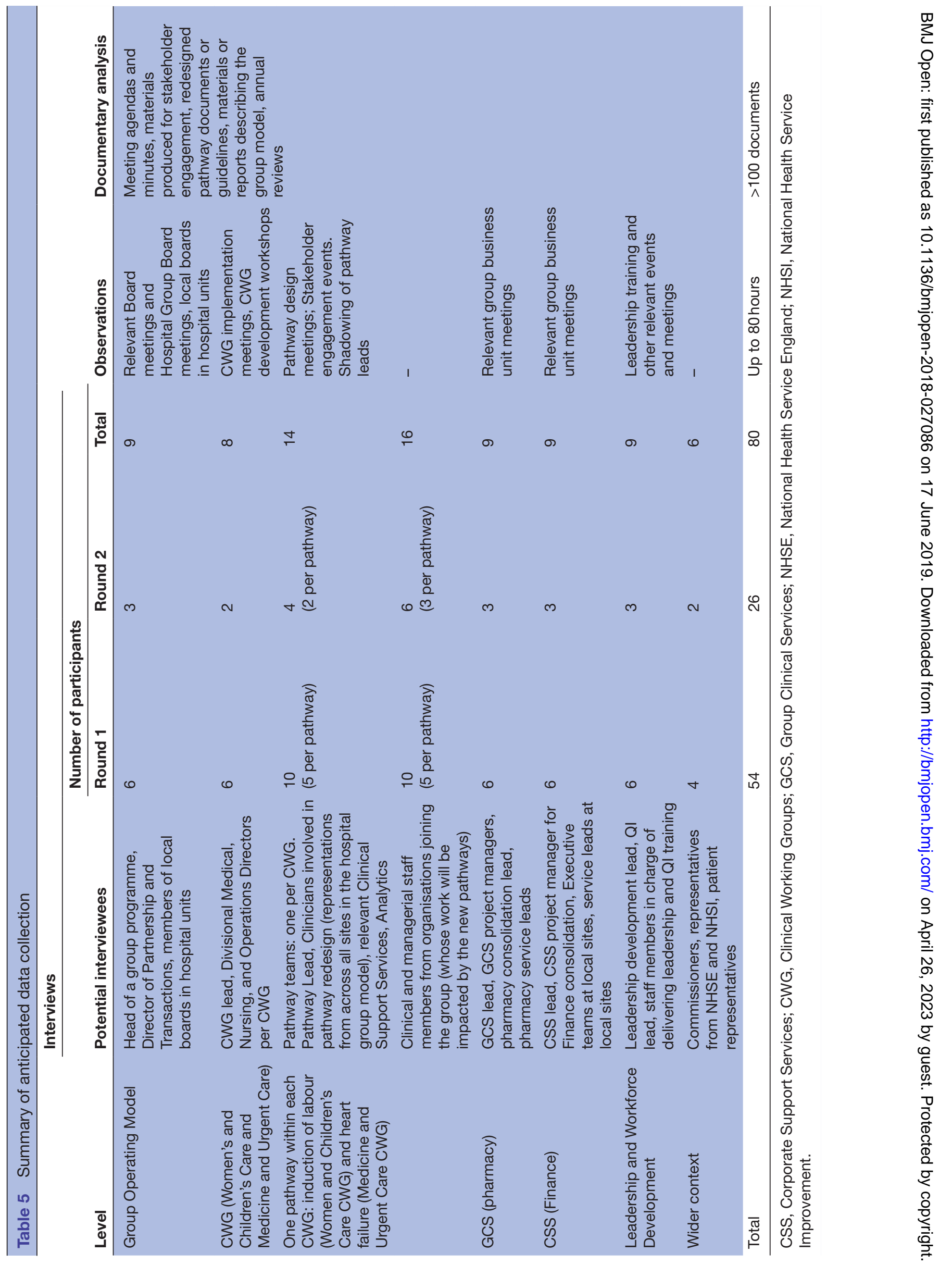


a day-to-day basis, in the areas selected for evaluation by organisations within the group will be obtained via documentary analysis. Second, data on time spent on each activity directly observed by the research team, including collaboration meetings, workshops and trainings, will be collected. For non-observed activities, an estimate of time and participating staff will be requested from the hospital group, in order to include staff opportunity time costs. The translation of time costs into monetary costs could be based on the Unit Costs for Health and Social Care, from the Personal Social Services Research Unit (PSSRU), or alternatively one could use the actual hourly costs of staff, should this information be available to the research team.

For the ROI analysis, we will require data on the volume and costs of corporate support and group-clinical services. For the selected CWG pathways, we will require data on length of stay, unexpected hospital admissions, unexpected intensive care unit (or neonatal intensive care unit) admissions, waste of materials, unnecessary use of medication/drugs and unnecessary surgical procedures (eg, non-emergency caesarean sections) covering 6-12 months before and 6-12 months after the transformation into the group model. For both selected CWG pathways, we will also require the total volume of activity, as well as the proportion of patients who are low and high risk, and those who end up having complications (by complication type). Data on the distribution of patients along the pathway is paramount for the ROI to be consistent and meaningful. The embedded research team will provide formative feedback to increase the likelihood of measuring the required data consistently across all group members. Should these data be unavailable for a full return on investment analysis, we will perform a simpler prospective analysis, using cost-savings parameters obtained in a literature review. This type of analysis would highlight the potential for cost-savings.

\section{Sampling framework}

Implementation study of the group model

We will sample staff members using the vertical slice technique, ${ }^{15} 16$ whereby staff members representing different layers, sites and sectors of the organisation are sampled purposively. We will also use snowball sampling ${ }^{17}$ to capture additional insights into planning and implementation, for instance, alternative perspectives on the changes (see table 5 for sampling framework). The snowball sampling will use data from the interviews and observations.

We will examine implementation and impact of Clinical Working Groups at different stages of development and across the different sites by tracking two pathways: induction of labour (Women's and Children's Care CWG) and heart failure (Medicine and Urgent Care CWG). These pathways were selected for the following reasons: (1) both pathways will involve all sites currently included in the hospital group model; (2) one pathway has undergone digitisation (induction of labour); (3) one pathway represents elective care, whereas the other pathway represents emergency care; (4) one pathway is more advanced in terms of development. These pathway characteristics are important because they will allow us to identify the factors that play a role at different stages of implementation of the pathways, the ways in which clinical context might shape implementation and the impact of technological innovations (ie, digitisation) on standardisation and the delivery of care. We will also analyse the development of the leadership and QI programmes and consolidation of clinical and non-clinical services as cross-cutting interventions involving multiple sectors of the organisations in the group model.

\section{Economic evaluation}

For the three areas included in the economic evaluation (table 4), the CCA (or CCA framework) will be informed by the organisational study. Therefore, no additional sampling is required. In turn, interviews are not necessary for the ROI analysis after the scoping stage, although this type of analysis requires sufficient longitudinal data, which will be requested from the selected CWGs and pathways.

\section{Data analysis}

Implementation study of the group model

Data analysis will be carried out in parallel to data collection to allow the sharing of interim findings. Iterative thematic analysis of interview, observation and documentary data will be conducted, following established procedures of constant comparative analysis. ${ }^{18}$ Validity will be assessed in relation to Patton's four criteria of validity in qualitative research: verification, rival explanations, negative cases and triangulation. ${ }^{19}$

Findings from the interviews and observations will be triangulated with relevant documents and local metrics (eg, metrics established locally to measure group performance, metrics on activity and outcomes for the new pathways). In terms of implementation, pathway teams have been identified within the hospital group's operating model as key to getting clinical standards into practice. By observing teams in action and shadowing pathway leads, we would aim to identify lessons on how the implementation is led in a range of clinical and organisational settings. We will also look at the processes used to design and deliver standardised clinical practice (considering the role of technology as a potential facilitator through digitisation). Documents will be analysed to further develop an understanding of the programme, the theory of improvement and how it changed, and track implementation of the programme over time.

\section{Health economic evaluation}

Following data collection and preliminary data analysis for the organisational study, qualitative findings will be used to develop the consequences for the CCA and these will mirror the objectives of the programme theory (table 2). The final number of consequences is yet to be determined. The most suitable consequence measures 
Table 6 Objectives, possible consequences and measurement scale

\begin{tabular}{lll}
\hline Objective & Consequence & Scale \\
\hline $\begin{array}{l}\text { Standardise processes to reduce } \\
\text { unwarranted variation }\end{array}$ & Percentage of standardised pathways & $\begin{array}{l}\text { Ratio between the number of pathways } \\
\text { standardised and total number of } \\
\text { pathways }\end{array}$ \\
$\begin{array}{lll}\text { Use leadership and expertise to drive } & \text { Increase in leadership training } & \text { Scale 1-4 for both consequences: } \\
\text { improvement } & \text { Increase in uptake of leadership training } & \text { 1: No increase } \\
& \text { and events } & \text { 2: Small increase } \\
& & \text { 3: Moderate increase } \\
& & \text { 4: Large increase }\end{array}$
\end{tabular}

$\begin{array}{ll}\text { Deliver system-wide benefits through } & \text { Reduction in admissions to secondary } \\ \text { whole pathway re-design } & \text { care (gap and ratio) }\end{array}$

Out of scope

Greyed out row (on whole pathway design or population health) outside the scope of this evaluation, as it is expected that system-wide benefits will take longer to realise than the 30 months of this evaluation.

will be determined jointly and discussed with relevant stakeholders, following the principle of co-production. The CCA will draw on some of the lessons learnt from the qualitative research, effectively producing a mixedmethods evaluation. Table 6 provides examples of possible consequence measures. For three areas of the evaluation, a separate CCA will be conducted (table 4).

The ROI data analysis will be carried out at different stages, given that it depends on the timing of implementation. ROI studies for the two selected pathways may require some time lag between implementation (and associated costs) of the (standardised) pathway and cost reductions, as it is expected that some effects will take longer to be observed. In order to assess the return on investment, data for all investments made and cost involved in the two pathways would be required before and after the implementation of the model.

\section{Combining the strands of the study}

This evaluation is designed to be mixed-methods, in which quantitative components and qualitative components are jointly analysed to provide a more comprehensive picture. Moreover, the ethos of embedded research and the principle of co-production help ensure that a formative feedback process enables the quantitative and qualitative components of the study to provide a joint narrative and substantiate joint findings.

The economic analysis will draw from the qualitative multisited ethnography component of the evaluation and learning shared throughout the evaluation to define and refine the consequences framework in the CCA, as well as to provide narrative reasoning for the emerging economic findings. Interviews will be used to inform the economic evaluation, including the collection of data on cost (eg, new staff roles and time allocated by existing staff). Although the final CCA will be produced at a later stage in the evaluation time frame, interim findings, particularly focused on progress and achievement (or the lack thereof) in terms of consequences will be shared regularly with the hospital group stakeholders. The qualitative study will use the interim findings from the economic evaluation to collect information that might be missing or target areas not originally included in the evaluation design. Once the qualitative emerging findings are turned into quantifiable consequences, a narrative from the ethnographic component of the evaluation will be used to explain changes in consequence outcomes.

\section{Patient and public involvement (PPI)}

We have set-up and coordinate an evaluation PPI panel comprising patient representatives, carer representatives and members of the public. Panel members have been involved since early stages of study design, providing input on the development of research questions, identification of areas of the group model to include in the evaluation, and the methods of data collection and analysis. The panel will be involved throughout the study.

\section{ETHICS AND DISSEMINATION}

The study protocol was reviewed by the local $\mathrm{R} \& \mathrm{D}$ office and the UCL Ethics Committee and classified as a service evaluation, thus not requiring approval by a research ethics committee. However, we will conduct this evaluation to the highest ethical standards, following guidelines for data security, confidentiality and information governance. We will follow an informed consent process with potential participants for the interviews and observations. We will maintain the anonymity of all research participants. Data will be stored securely and only members of the team will have access.

The successful implementation of the evaluation will depend on ensuring the researchers have access to people, areas and data. The areas covered in the evaluation will depend on their implementation within the study time frame. The relationships created and maintained with staff throughout the evaluation will be instrumental in guaranteeing this access. Another potential challenge of embedded research is the loss of impartiality. ${ }^{7}$ Importantly, whereas the embedded team will work in close partnership with professionals and managers, the team will retain critical distance in order for the evaluation 
to provide independent findings. Our research team has previous experience of working in similar situations and has established a series of strategies to address these potential tensions between co-production and independent research: (1) although one of the researchers is embedded, the rest of the research team is based at UCL, providing a more distant and critical perspective; (2) the UCL team and staff from healthcare organisations will agree during initial stages of the project and throughout that the UCL team will maintain an independent role throughout the evaluation, will share both positive and negative feedback to different groups of stakeholders within the organisation and will publish the findings.

The team will share feedback on a quarterly basis with relevant Boards and other groups across the organisation (including patient groups). These dissemination arrangements will be reviewed regularly with stakeholders over the course of the study. Formative feedback will include ${ }^{1}$ sharing our evolving understanding of the programme theory and suggested refinements'; providing 'realtime' insights into implementation of new structures (eg, CWGs) that are being used to enable organisational change (eg, areas that are being neglected, factors that are acting as barriers in implementation and potential solutions, and groups that might have been excluded); and $^{3}$ analysing staff views on the process of change.

The evaluation will also generate wider lessons for the NHS to support providers planning new organisational models, including guidance for potential adopters of new models of care on implementing change. Our embedded research approach will allow us to be responsive to the hospital group's needs, so we can adapt the frequency and format of feedback in line with the organisation's requirements. We will also aim to disseminate the study findings through articles published in peer-reviewed journals and presentations in academic conferences.

Contributors All contributors participated in the design of the study. CV-P and ECB: drafting of the manuscript. CV-P, ECB, AIGR, ST, RA, AC-N, SM, NJF: made significant contributions to the manuscript and approved the final version before submission.

Funding This work was supported by NHS England. ECB, NJF and SM were supported by the National Institute for Health Research (NIHR) Collaboration for Leadership in Applied Health Research and Care North Thames at Bart's Health NHS Trust (NIHR CLAHRC North Thames). NJF is an NIHR Senior Investigator.

Disclaimer The views expressed in this article are those of the author(s) and not necessarily those of the NHS, the NIHR, or the Department of Health and Social Care.
Competing interests RA and ACN are staff members from the hospitals that are part of this evaluation and contribute to the planning and implementation of the hospital group model. CV-P is working as an embedded researcher throughout the course of the evaluation.

Patient consent for publication Not required.

Provenance and peer review Not commissioned; externally peer reviewed.

Open access This is an open access article distributed in accordance with the Creative Commons Attribution Non Commercial (CC BY-NC 4.0) license, which permits others to distribute, remix, adapt, build upon this work non-commercially, and license their derivative works on different terms, provided the original work is properly cited, appropriate credit is given, any changes made indicated, and the use is non-commercial. See: http://creativecommons.org/licenses/by-nc/4.0/.

\section{REFERENCES}

1. NHS England. No hospital is an island: Learning from the Acute Care Collaboration vanguards, 2018.

2. NHS England, Public Health England, Care Quality Commission, Trust Development Authority, Monitor HEE. Five Year Forward View, 2017.

3. Department of Health. Examining new options and opportunities for providers of NHS care: The Dalton Review, 2014.

4. Consulting C. NHS Group Models: Working together for a more sustainable NHS, 2017.

5. The King's Fund. Perspectives for the Dalton review: Future organisational models for the NHS, 2014.

6. RFL. The Royal Free London NHS Foundation Trust Value Propostion for FY17, 2017.

7. Vindrola-Padros $\mathrm{C}$, Pape $\mathrm{T}$, Utley $\mathrm{M}$, et al. The role of embedded research in quality improvement: a narrative review. BMJ Qual Saf 2017;26:70-80.

8. Marshall M, Pagel C, French C, et al. Moving improvement research closer to practice: the Researcher-in-Residence model. BMJ Qual Saf 2014;23:801-5.

9. Ferlie E, Fitzgerald L, McGIVERN G, et al. Public policy networks and 'wicked problems': A nascent solution? Public Adm 2011;89:307-24.

10. Marcus GE. Ethnography in/of the World System: The Emergence of Multi-Sited Ethnography. Annu Rev Anthropol 1995;24:95-117.

11. Marcus G. Multi-sited ethnography: Notes and queries. Falzon M, ed. Multi-sited Ethnography: Theory, Praxis and Locality in Contemporary Research. London (UK): Routledge, 2016.

12. Drummond MF, Sculpher MJ, Claxton K, et al; Methods for the economic evaluation of health care programmes. Oxford University Press: Oxford, 2015.

13. Greenhalgh T. How to read a paper. Papers that tell you what things cost (economic analyses). BMJ 1997;315:596-9.

14. Knoblauch H. Focused ethnography. Forum Qual Soc Res 2005;6.

15. Nader L. The vertical slice: Hierarchies and children. In: Britan G, Cohen R, eds. Hierarchy and society: Anthropological perspectives on society. Philadelphia, PA: Institute for the Study of Human Issues, 1980.

16. Gonzalez R, Stryker R. On studying up, down, and sideways: What's at stake?: In. Up, down and sideways: Anthropologists trace the pathways of power.. New York: Berghahn Books, 2014.

17. Noy C. Sampling knowledge: The hermeneutics of snowball sampling in qualitative research. Int J Soc Res Methodol 2008;11:327-44.

18. Mays N, Pope C. Qualitative research: Rigour and qualitative research. BMJ 1995;311:109-12.

19. Patton M. Qualitative evaluation and research methods. SAGE 2002. 